\title{
DiHS/DRESS syndrome induced by second-line treatment for tuberculosis and Epstein-Barr virus
}

\author{
IEVA BAJORIUNIENE ${ }^{l}$, GRETA MUSTEIKIENE ${ }^{2}$, AGNE RAMONAITE ${ }^{1}$, BRIGITA SITKAUSKIENE ${ }^{l}$
}

${ }^{1}$ Department of Immunology and Allergology, Medical Academy, Lithuanian University of Health Sciences, Kaunas, Lithuania ${ }^{2}$ Department of Pulmonology, Medical Academy, Lithuanian University of Health Sciences, Kaunas, Lithuania

\begin{abstract}
Drug-induced hypersensitivity syndrome (DiHS) or drug reaction with eosinophilia and systemic symptoms (DRESS) syndrome is a severe adverse drug-induced reaction characterized by various symptoms: skin rash, fever, lymph node enlargement and internal organ involvement, which starts within 2 weeks to 3 months after drug initiation. It is challenging to diagnose this syndrome due to the variety of cutaneous and visceral symptoms. Different mechanisms have been implicated in its development, including genetic susceptibility associated with human leucocyte antigen (HLA) loci, detoxification defects leading to reactive metabolite formation and subsequent immunological reactions, slow acetylation, and reactivation of human herpes, including Epstein-Barr virus and human herpes virus (HHV)-6 and HHV-7. The most frequently reported causes of DiHS/DRESS are antiepileptic agents, allopurinol and sulfonamides. We report a case of DiHS/DRESS induced by second-line treatment for tuberculosis, prothionamide and para-aminosalicylic acid, and Epstein-Barr virus re-infection. Patch testing, which was performed in this case, is not fully standardized, but it can be helpful and a safe way to evaluate and diagnose DiHS/DRESS.
\end{abstract}

Key words: drug-induced hypersensitivity syndrome, drug reaction with eosinophilia and systemic symptoms, second-line treatment for tuberculosis.

(Cent Eur J Immunol 2021; 46 (3): 401-404)

\section{Introduction}

Drug-induced hypersensitivity syndrome (DiHS) or drug reaction with eosinophilia and systemic symptoms (DRESS) syndrome is a rare and severe hypersensitivity reaction to a drug that includes skin eruption, hematologic abnormalities (eosinophilia, atypical lymphocytes), lymphadenopathy, and internal organ involvement (liver, kidney, lung, etc.) [1, 2]. DiHS/DRESS syndrome is characterized by a long latency ( 2 to 8 weeks) after the initiation of drug therapy and the possible persistence or aggravation of symptoms despite the discontinuation of the culprit drug, and frequent association with the reactivation of a latent human herpes virus (HHV) or other infection [3]. The pathogenesis of DiHS/DRESS syndrome is understood partially. Different mechanisms have been implicated in its development, including genetic susceptibility associated with human leucocyte antigen (HLA) loci, detoxification defects leading to reactive metabolite formation and subsequent immunological reactions, slow acetylation, and reactivation of human herpes, including Epstein-Barr virus and HHV-6 and HHV-7 [4, 5]. We report a case of DiHS/ DRESS induced by second-line treatment for tuberculosis and Epstein-Barr virus re-infection.

\section{Case report}

A 31-year-old woman was admitted to the hospital for the treatment of newly diagnosed drug-resistant (pre-extensively drug resistant) pulmonary tuberculosis (chest X-ray at time of diagnosis - Fig. 1). She was treated with second-line antituberculosis drugs: moxifloxacin, kanamycin, cycloserine, prothionamide, para-aminosalicylic acid. After 3 weeks of therapy she developed high fever $\left(>39^{\circ} \mathrm{C}\right)$, lymphadenopathy in the cervical and axillary regions and pruritic maculopapular eruption all over the body (Fig. 2). Hematologic abnormalities such as leukocytosis with eosinophilia $\left(1.81 \times 10^{9} / 1\right)$ and monocytosis $\left(1.85 \times 10^{9} / 1\right)$ were detected in peripheral blood of the patient. Hepatitis was asymptomatic and detected by the evaluation of liver function: serum aspartate aminotransferase (AST) $1379 \mathrm{IU} / \mathrm{l}$ and alanine aminotransferase (ALT) $1221 \mathrm{IU} / \mathrm{l}$; levels of liver enzymes were increased by approximately 30-40-fold above the normal limits. The positive diagnosis of Epstein-Barr infection was based on the onset of increase in the anti-Epstein-Barr immunoglobulin (Ig) $\mathrm{G}$ titer (> $200 \mathrm{U} / \mathrm{ml}$ ), implicating Epstein-Barr virus re-activation. Based on the clinic and laboratory findings diagnosis of DiHS/DRESS was suspected, and all the drugs were dis-

Correspondence: Dr. Ieva Bajoriuniene, Department of Immunology and Allergology, Medical Academy, Lithuanian University of Health Sciences, LT 44307 Kaunas, Lithuania, e-mail: ieva.bajoriuniene@1smuni.lt Submitted: 16.09.2020; Accepted: 10.05.2021 

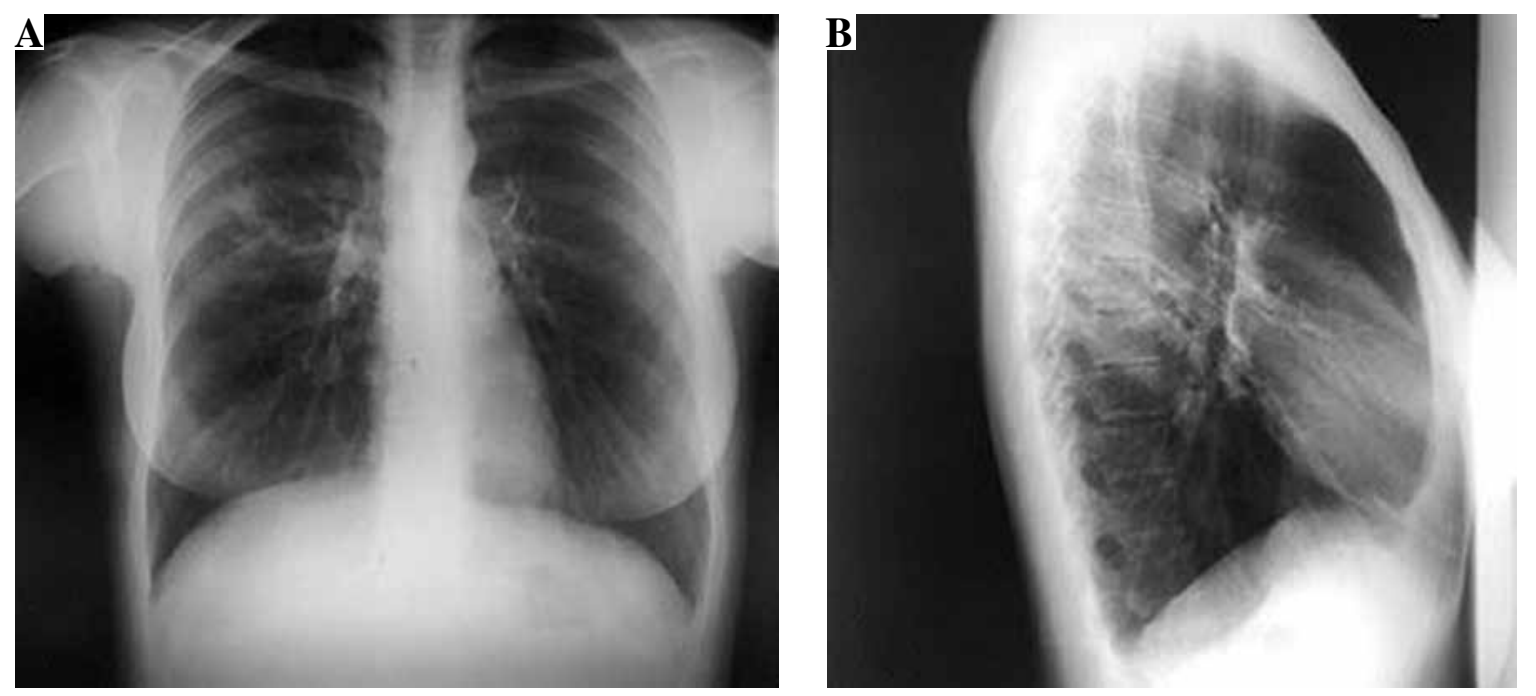

Fig. 1. Chest $X$ ray posterior-anterior view (A) and right lateral view (B) on admission. On the superior lobe posterior segment of the right lung a mild parenchymal opacity with several nodules can be seen
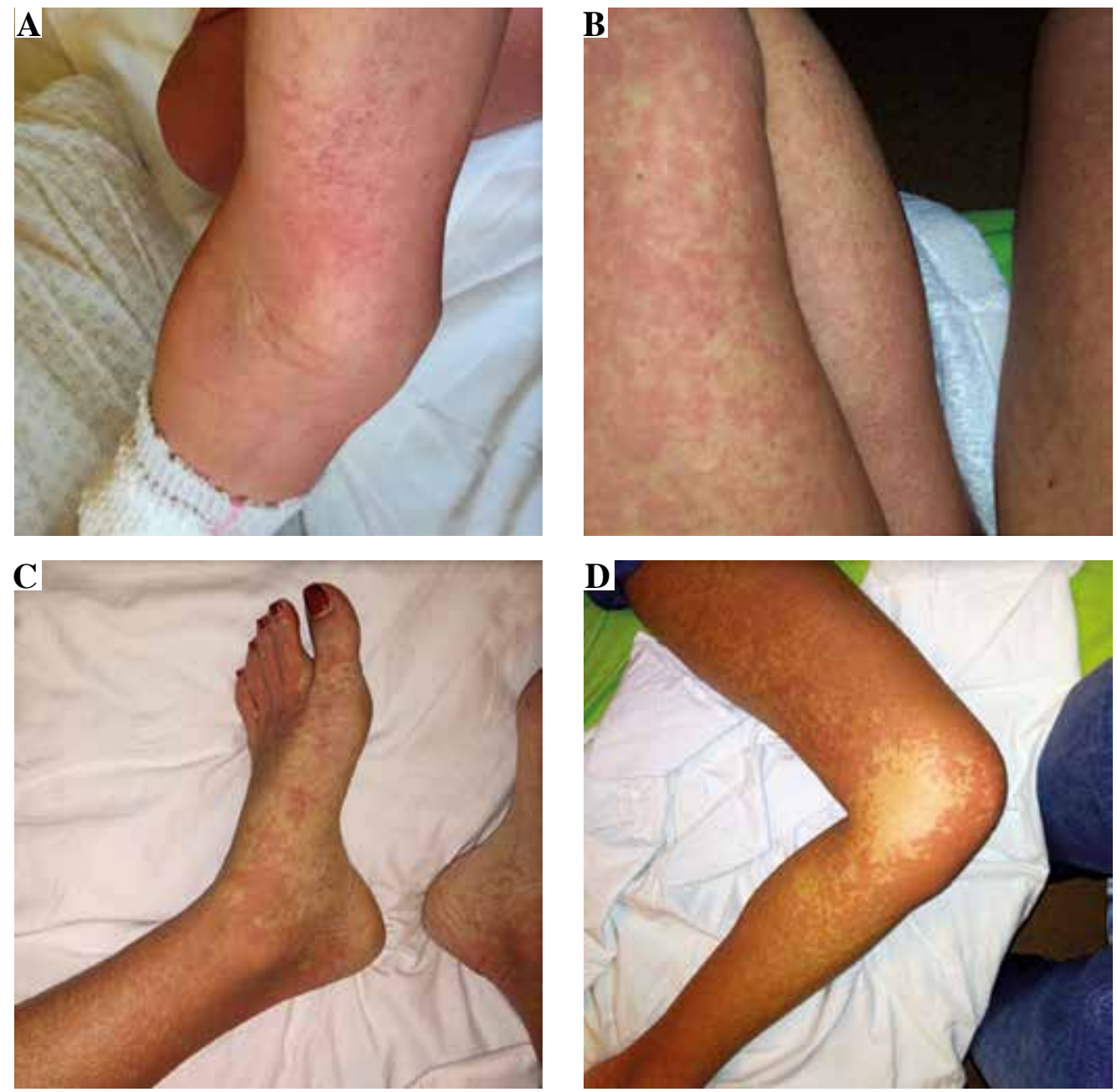

Fig. 2. Macculopapular exanthema on the patients body after three weeks of second-line treatment for tuberculosis 
Table 1. Patch test was performed 4 weeks after the hypersensitivity syndrome resolved

\begin{tabular}{lcc}
\hline Evaluation & After 48 h & After 72 h \\
\hline Moxifloxacin 30\% pet. & - & - \\
\hline Kanamycin 30\% pet. & - & - \\
\hline Cycloserine 30\% pet. & - & - \\
\hline Prothionamide 30\% pet. & + & + \\
\hline Para-aminosalicylic acid 30\% pet. & ++ & ++ \\
\hline
\end{tabular}

pet-petrolatum, interpretation of the test results [according to the International Contact Dermatitis Research Group (ICDRG)]: - negative reaction, + weak positive reaction, ++ strong positive reaction.

continued. Symptoms and laboratory abnormalities gradually resolved over 4 weeks without additional treatment.

A patch test was performed [6] and analyzed [7] according to the recommendations. The results of the patch test are presented in Table 1 and Figure 3. Treatment was adjusted to ethambutol, kanamycin, cycloserine, pyrazinamide, and linezolid, and no new symptoms were observed.

\section{Discussion}

DiHS/DRESS syndrome is a severe adverse reaction to a drug characterized by various symptoms - skin rash, fever, lymph node enlargement and internal organ involvement - which starts within 2 weeks to 3 months after drug initiation. The pathogenesis of DiHS/DRESS is not fully understood, but it is associated with a delayed immunological reaction to a drug $[1,8]$. Genetic polymorphism (HLA) and HHV reactivation may also cause DiHS/DRESS syndrome. The death rate is about $10 \%$, mostly due to liver damage thought to be mediated by infiltration of eosinophils [9]. The most frequently reported causes of DiHS/
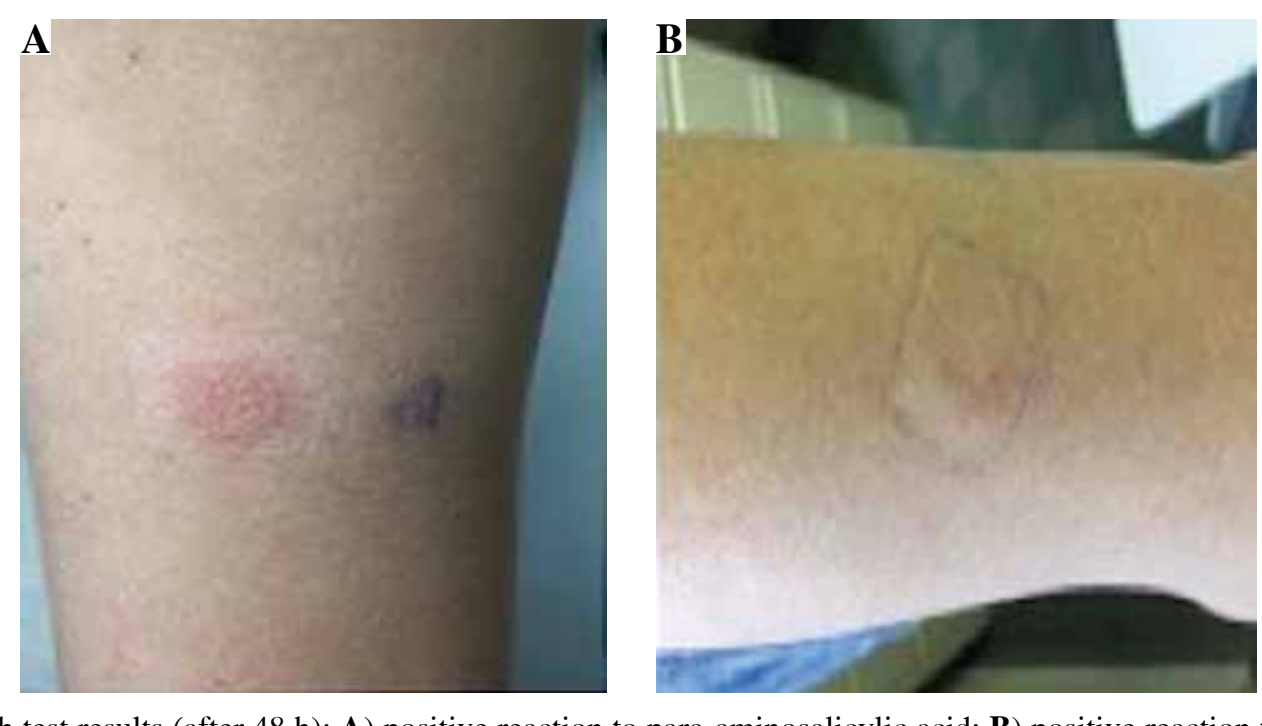

Fig. 3. Patch test results (after $48 \mathrm{~h}$ ): A) positive reaction to para-aminosalicylic acid; B) positive reaction to prothion-

amide

DRESS are antiepileptic agents, allopurinol, sulfonamides and dapsone [10]. Anti-tuberculosis drugs may also induce DiHS/DRESS syndrome [11]; according to the literature, rifampicin was the most commonly suspected drug, followed by isoniazid, ethambutol and pyrazinamide [12].

PubMed search showed only a few case reports of DiHS/DRESS induced by the second line anti-tuberculosis drugs, such as prothionamide and para-aminosalicylic acid [13]. The drug provocation test is generally accepted as a gold standard for confirmation of the culprit drug [14] and may minimize anti-tuberculosis treatment interruption; however, in severe cases, such as the currently described case, the provocation test is not ethically recommended. Furthermore, in a case which involves multiple complexed treatment, the patch test may be a useful method for detection of the culprit drug, as we applied in our case. According to the guidelines for performing patch tests in drug-induced cutaneous adverse reactions, tests should be performed 6 months after the complete healing of DiHS/ DRESS $[6,15]$. However, previous patch tests could be justified because of the nature of a tuberculosis infection, the lack of adequate therapeutic alternatives, and the risk/ benefit balance. A positive test result is highly dependent on the causative drug. We tested all suspected drugs incorporated at $30 \%$ in white petrolatum (pet.) [6]. Our patient's patch test was positive to prothionamide and para-aminosalicylic acid. The analysis of patch test results was difficult because it was performed relatively close to the acute phase and in the context of immunosuppression due to tuberculosis infection. According to the ENDA/EAACI Drug Allergy Interest Group position paper, it might be advisable to perform the lymphocyte transformation test (LTT) before in vivo tests in severe reactions with a suspected T cell mechanism [16]. However, positive LTT reactions can only be obtained at the recovery phase, 2 months after 
onset; thus negative LTT reactions at the acute phase could alternatively be interpreted as suggesting a diagnosis of DiHS/DRESS [17].

Recently, Epstein-Barr virus, HHV-6, and HHV-7 reactivations were found in $76 \%$ of DiHS/DRESS cases in a clinical study. Interestingly, the culprit drugs were able to trigger viral reactivations that induce a pathogenic antiviral $\mathrm{T}$ cell immune response [18]. In our case, Epstein-Barr laboratory tests showed IgG antibody positive reaction, but the PCR assay to detect the virus was not done. Hence an association of DiHS/DRESS with Epstein-Barr virus infection cannot be fully confirmed.

In conclusion, the diagnosis of DiHS/DRESS should be highly suspected with the presence of skin rash, liver involvement, fever, eosinophilia, and lymphadenopathy. The list of drugs causing DiHS/DRESS syndrome should be expanded with second-line drugs for tuberculosis treatment such as prothionamide and para-aminosalicylic acid. The high rate of herpes viruses reactivation associated with DiHS/DRESS implies that these viruses should be detected in all suspected cases during daily clinical practice. Additionally, clinicians who prescribe treatment for tuberculosis must be aware of the possibility of a severe hypersensitivity reaction to anti-tuberculosis drugs.

\section{The authors declare no conflict of interest.}

\section{References}

1. Cacoub P, Musette P, Descamps V, et al. (2011): The DRESS syndrome: a literature review. Am J Med 124: 588-597.

2. Bocquet H, Bagot M, Roujeau JC (1996): Drug-induced pseudolymphoma and drug hypersensitivity syndrome (Drug Rash with Eosinophilia and Systemic Symptoms: DRESS). Semin Cutan Med Surg 15: 250-257.

3. Picard D, Janela B, Descamps V, et al. (2010): Drug reaction with eosinophilia and systemic symptoms (DRESS): a multiorgan antiviral T cell response. Sci Transl Med 46: 46ra62.

4. Nanishi E, Hoshina T, Ohga S, et al. (2015): Drug reaction with eosinophilia and systemic symptoms during primary Epstein-Barr virus infection. J Microbiol Immunol Infect 48: 109-112.

5. Camous X, Calbo S, Picard D, et al. (2012): Drug reaction with eosinophilia and systemic symptoms: an update on pathogenesis. Curr Opin Immunol 24: 730-735.

6. Barbaud A, Gonçalo M, Bruynzeel D, et al. (2001): European Society of Contact Dermatitis. Guidelines for performing skin tests with drugs in the investigation of cutaneous adverse drug reactions. Contact Dermatitis 45: 321-328.

7. Fonacier L (2015): A practical guide to patch testing. J Allergy Clin Immunol Pract 3: 669-675.

8. Hansel K, Bellini V, Bianchi L, et al. (2017): Drug reaction with eosinophilia and systemic symptoms from ceftriaxone confirmed by positive patch test: An immunohistochemical study. J Allergy Clin Immunol Pract 5: 808-810.

9. Chen YC, Chiu HC, Chu CY (2010): Drug reaction with eosinophilia and systemic symptoms: a retrospective study of 60 cases. Arch Dermatol 46: 1373-1379.
10. Kardaun SH, Sekula P, Valeyrie-Allanore L, et al. (2013): Drug reaction with eosinophilia and systemic symptoms (DRESS): an original multisystem adverse drug reaction. Results from the prospective RegiSCAR study. Br J Dermatol 169: 1071-1080.

11. Kim SH, Lee SK, Kim SH, et al. (2013): Antituberculosis drug-induced hypersensitivity syndrome and its association with human leukocyte antigen. Tuberculosis (Edinb) 93: 270274.

12. Allouchery M, Logerot S, Cottin J, et al. (2018): Antituberculosis drug-associated DRESS: a case series. J Allergy Clin Immunol Pract 6: 1373-1380.

13. Kim JH, Jang SH, Kim DH, et al. (2013): A case of DRESS syndrome induced by the antituberculosis drugs, prothionamide, and para-aminosalycilic acid. Ann Allergy Asthma Immunol 110: 118-119.

14. Aberer W, Bircher A, Romano A, et al. (2003): Drug provocation testing in the diagnosis of drug hypersensitivity reactions: general considerations. Allergy 58: 854-863.

15. Santiago F, Gonçalo M, Vieira R, et al. (2010): Epicutaneous patch testing in drug hypersensitivity syndrome (DRESS). Contact Dermatitis 62: 47-53.

16. Mayorga C, Celik G, Rouzaire P, et al. (2016): In vitro tests for drug hypersensitivity reactions: an ENDA/EAACI Drug Allergy Interest Group position paper. Allergy 71: 1103-1134.

17. Shiohara T, Mizukawa Y (2019): Drug-induced hypersensitivity syndrome (DiHS)/drug reaction with eosinophilia and systemic symptoms (DRESS): An update in 2019. Allergol Int 68: 301-308.

18. Gentile I, Talamo M, Borgia G (2010): Is the drug-induced hypersensitivity syndrome (DIHS) due to human herpesvirus 6 infection or to allergy-mediated viral reactivation? Report of a case and literature review. BMC Infect Dis 10: 49. 